\title{
Smartphysics: el uso docente del Smartphone para el desarrollo de competencias transversales
}

\author{
J.Á. Sans, V.P. Cuenca-Gotor, F.J. Manjón, I. Salinas, M.H. Gimenéz, J.A. Monsoriu, \\ J.A. Gómez-Tejedor \\ ETS de Ingeniería del Diseño, Universitat Politècnica de València. jogomez@fís.upv.es
}

\begin{abstract}
In this paper, we propose to introduce new physics laboratory practices and modify some ones, with the main objective of developing in our students some of the transversal competences established by the Universitat Politècnica de València (UPV), such as critical and practical thinking, understanding and integration, lifelong learning, effective communication, teamwork and leadership. The instrument used to achieve these goals is the smartphone, employed both as a measuring device and as a data recorder. We think the use of the smartphone will be a motivator element for the student since they are used to it and they will have the opportunity to explore new applications of this device.
\end{abstract}

Keywords: Smartphone, Smartphone Sensors, Physics Laboratory, New Technologies, Transverse Competences.

\begin{abstract}
Resumen
En este trabajo proponemos introducir nuevas prácticas de laboratorio de física, y modificar algunas existentes, con el objetivo principal de que nuestros estudiantes desarrollen algunas de las competencias transversales establecidas por la Universitat Politècnica de València (UPV), tales como pensamiento crítico, comprensión e integración, aplicación pensamiento práctico, aprendizaje permanente, comunicación efectiva, y trabajo en equipo y liderazgo. El instrumento que vamos a utilizar para alcanzar este objetivo es el teléfono móvil inteligente (smartphone), que operará tanto como dispositivo de medida como de almacenamiento de datos. Creemos que el uso del teléfono móvil será un elemento motivador para el alumno ya que están acostumbrados a su uso y además tendrán la oportunidad de conocer nuevas aplicaciones de este dispositivo.
\end{abstract}

Palabras clave: Smartphone, Sensores del teléfono móvil, Laboratorio de Física, Aplicación de Nuevas Tecnologías, Competencias Transversales. 


\section{Introducción}

En el laboratorio de física se realizan prácticas de laboratorio en las cuales los alumnos estudian desde el punto de vista experimental leyes físicas que han trabajado previamente en las clases de teoría. El objetivo de las prácticas de laboratorio es doble: por un lado reforzar los conocimientos teóricos adquiridos en las clases de teoría, y por otro lado habituarse a las técnicas experimentales propias de laboratorio: manejo de aparatos de medida, toma de datos experimentales, análisis de datos experimentales, cálculos de errores, etc.

En el laboratorio de física se utilizan multitud de aparatos de medida. Algunos son muy sencillos (cronómetro, regla) y otros son mucho más complejos (multímetro, osciloscopio, ordenador). Es muy importante para la correcta formación del alumno de grados tecnológicos, que adquiera habilidades en la utilización de dichos instrumentos de medida así como en el análisis matemático de los datos obtenidos.

Nuestra experiencia docente ha revelado cómo, en ocasiones, los alumnos encuentran rutinarias y poco motivadoras las prácticas de las asignaturas relacionadas con la física. Esto conlleva falta de interés en las mismas y que traten de realizar en el mínimo tiempo posible el número mínimo de medidas exigidas por el profesor para poder terminar rápidamente, de una manera mecánica, y sin reflexión crítica sobre el trabajo realizado. Por otro lado, el uso de material antiguo y/o ajeno al uso cotidiano del alumno aleja a éste de la propia experiencia.

Desde hace algún tiempo, algunos profesores del departamento de física en la Escuela Técnica Superior de Ingeniería del Diseño estamos trabajando en la utilización del smartphone como elemento motivador, tratando de integrarlo en las prácticas como dispositivo de medida y toma de datos a través de los sensores que incorpora. El Smartphone es un dispositivo que reúne las características de sencillez de operatividad, alto grado de sofisticación y uso atractivo, que permiten su utilización en el laboratorio de física de primeros cursos de universidad.

Los alumnos están acostumbrados a utilizar el smartphone en su vida social principalmente como objeto para comunicación y entretenimiento y en menor medida para sus estudios. En este trabajo pretendemos enseñar a los estudiantes de que el teléfono móvil puede ser útil también como dispositivo de medida y despertar su curiosidad e interés por utilizar este instrumento para diversas aplicaciones prácticas

En la literatura científica del área de docencia de la física se pueden encontrar propuestas de experimentos con smartphones. Estas propuestas están en la misma línea que este trabajo, pero ninguna de ellas hace referencia a la implementación de estos experimentos en grupos de alumnos (Klein et al. 2014; Kuhn y Vogt 2012; González y González Rebollo 2015; Huete et al. 2015; Arribas Garde et al. 2015; Kuhn y Vogt 2013; Sans et al. 2013; CastroPalacio et al. 2013; Tuset-Sanchis et al. 2015; Gómez-Tejedor, Castro-Palacio y Monsoriu 2014; Castro-Palacio et al. 2014). Solamente en algunos trabajos recientes realizados por nuestro grupo, aparecen las primeras referencias a la implementación de este tipo de experiencias con alumnos (Sans et al. 2016; Cuenca-Gotor et al. 2015; Sans et al. 2015). 


\section{Objetivos}

El objetivo fundamental de la propuesta es introducir nuevas prácticas de laboratorio de física, y modificar algunas existentes, para que nuestros estudiantes desarrollen algunas de las competencias transversales establecidas por la UPV (UPV Vicerrectorado de Estudios Calidad y Acreditación 2016). Para ello, dados los buenos resultados obtenidos en trabajos anteriores, vamos a utilizar el smartphone como dispositivo de medida, de tal forma que los alumnos descubran que este dispositivo tecnológico puede tener muchas más aplicaciones de las que ellos conocen. En concreto, se pretende que los alumnos utilicen un smartphone de gama media (no es necesario uno de gama alta) para la medida de determinadas magnitudes físicas. Las prestaciones de los smartphones actuales permiten, además, que dichas magnitudes se registren y exporten a un ordenador para su posterior análisis, tal como se hace habitualmente en un laboratorio de física convencional.

En este aspecto, nos gustaría recalcar que en ningún momento planteamos sustituir los aparatos clásicos de medida por el smartphone ya que los sensores de los smartphones presentan ciertas limitaciones en cuanto a sensibilidad, error de cero y calibración de escala que dependen de las diferentes marcas y modelos. Nuestra idea es complementar las medidas que realizan los alumnos con los aparatos clásicos con la información que pueda aportar en esas medidas el propio smartphone del alumno. De esta forma los alumnos pueden realizar un análisis de los datos mucho más elaborado, porque les permite calcular una misma magnitud física con métodos diferentes, comparar los resultados, analizar los errores de cada método, discutir las diferencias y tratar de concluir cuál es el método más adecuado. Asimismo, los alumnos tienen que desarrollar un pensamiento crítico para entender los fundamentos en los que se basa la experiencia, comparar los resultados, y analizar razonadamente los resultados obtenidos, lo cual permite el desarrollo de la Competencia Transversal 9 (CT9) "pensamiento crítico". Además, los alumnos deben de demostrar la comprensión e integración del conocimiento tanto de la propia asignatura como en otros contextos más amplios, desarrollando así la CT1 "comprensión e integración".

En las prácticas de laboratorio, los alumnos aplican los conocimientos adquiridos en las clases teóricas a la práctica, atendiendo a la información disponible, y estableciendo el proceso a seguir para alcanzar los objetivos con eficacia y eficiencia, guiados en todo momento por el profesor, de modo que se trabaja la CT2 "aplicación pensamiento práctico". Es más, esta competencia se desarrolla especialmente ya que los alumnos utilizan de forma práctica e ingeniosa el Smartphone y sus sensores para aplicaciones para las que en principio no están diseñados.

Este proyecto fomenta la CT11 "aprendizaje permanente" de los alumnos, al dotarlos de una herramienta que les permita realizar sus propias experiencias científicas fuera de la Universidad de manera autónoma y flexible, en contraposición a los aparatos clásicos de medida que normalmente no están a su alcance. En particular, el uso del teléfono móvil es un ejemplo más del proceso de miniaturización que afecta a muchas aplicaciones

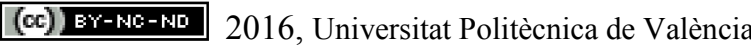

Congreso In-Red (2016) 
tecnológicas en su paso desde el laboratorio de investigación (en estudios ex situ fuera del ámbito de trabajo) al campo de trabajo, donde pueden aplicarse en el estudio in situ de procesos en tiempo real. Esto requiere que el alumno investigue, e incluso desarrolle, posibles aplicaciones que permitan el uso de los diferentes sensores que incorpora el Smartphone.

Para la evaluación de las prácticas de laboratorio los alumnos deben de presentar un informe escrito o "memoria de laboratorio" gracias al cual trabajan la CT8 "comunicación efectiva", teniendo que utilizar adecuadamente los recursos necesarios y adaptándose a las características de la situación. En esta memoria los alumnos deben de explicar el experimento realizado con su fundamento teórico; mostrar los resultados obtenidos y los cálculos realizados; así como las conclusiones obtenidas del experimento. Así pues, la realización de la memoria incide a su vez en las CT1 "comprensión e integración" y CT9 "pensamiento crítico". Este trabajo se realiza por equipos para la consecución de objetivos comunes, contribuyendo al desarrollo personal y académico de los miembros de estos equipos, desarrollando por tanto la CT6 "trabajo en equipo y liderazgo".

Estas competencias transversales serán evaluadas mediante la utilización de rúbricas para la calificación de las memorias de laboratorio, con la incorporación de apartados específicos para evaluar dichas competencias. La rúbrica es una "herramienta de evaluación que establece unos niveles estándar para medir la calidad de cada uno de los diferentes criterios con los que se puede desarrollar un objetivo, una competencia, un contenido o cualquier otro tipo de tarea que se lleve a cabo en el proceso de aprendizaje" (Goodrich Andrade 1996).

La evaluación de competencias transversales implica que la persona evaluada se enfrente al reto de la integración y utilización de conocimientos, destrezas técnicas, estrategias y actitudes para afrontar o resolver de manera apropiada una situación o problema propio de su ámbito profesional o académico. La rúbrica permite además la retroalimentación constructiva y crítica, tanto del aprendizaje como del proceso formativo, y fomenta la reflexión del trabajo realizado.

\section{Desarrollo de la innovación}

En este trabajo presentamos las siguientes prácticas de laboratorio mediante la utilización del Smartphone como dispositivo de medida y adquisición de datos:

\section{Oscilaciones: determinación de la constante de un muelle}

Tradicionalmente, los alumnos estudian el movimiento armónico simple mediante un muelle en el cual se sitúa una masa que se hace oscilar. Midiendo el período de la oscilación, los alumnos determinan la constante elástica del muelle.

Mediante el acelerómetro incorporado en el teléfono, se puede registrar la aceleración, en función del tiempo, del teléfono móvil suspendido de un muelle (Castro-Palacio et al. 2013; Tuset-Sanchis et al. 2015; Cuenca-Gotor et al. 2015; Castro-Palacio et al. 2014). De esta forma, los alumnos tienen una información mucho más rica (posición y/o aceleración en 
función del tiempo) para el estudio del movimiento armónico simple. Pueden calcular así el período, y compararlo con el que se obtiene de forma tradicional mediante la medida del tiempo de las oscilaciones (Sans et al. 2016; Cuenca-Gotor et al. 2015; Sans et al. 2015). En la figura 1 se muestra una fotografía de la sesión de prácticas del muelle, y en la figura 2 se muestra con más detalle la colocación del teléfono móvil para la realización de la práctica.

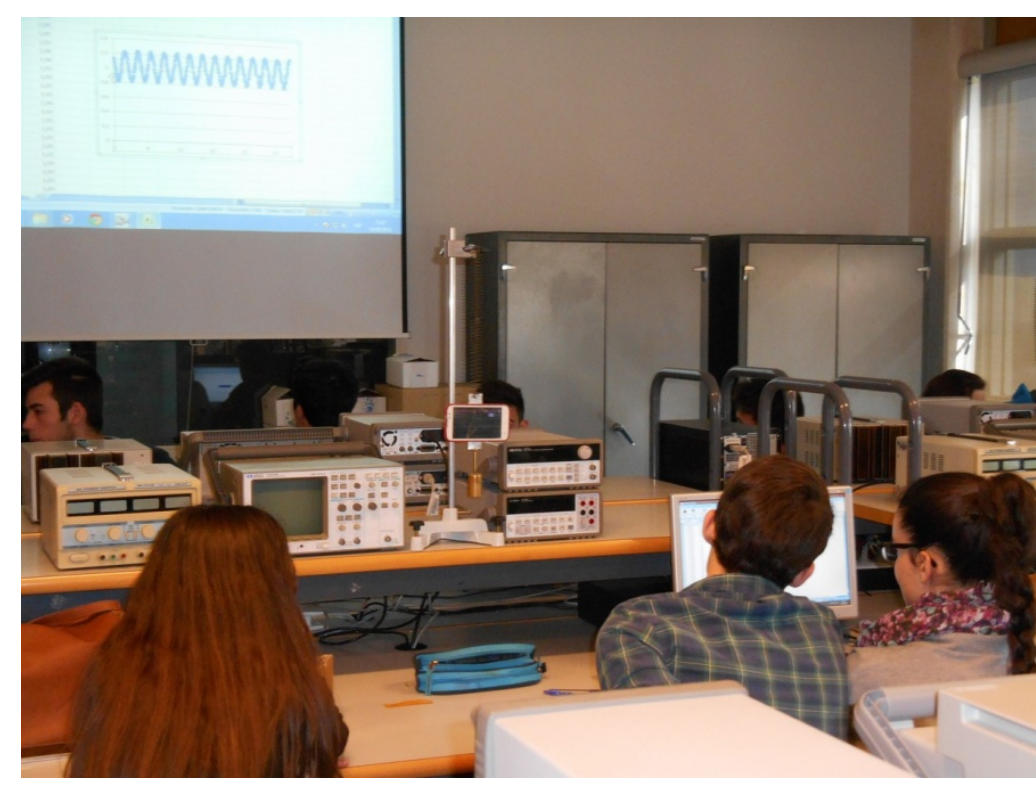

Fig. 1 Sesión de la práctica de Oscilaciones realizada con el teléfono móvil

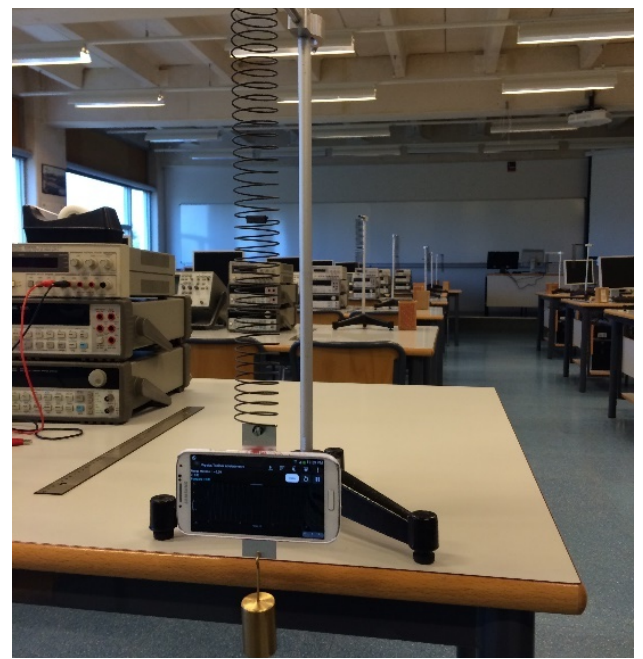

Fig. 2 Detalle de la colocación del teléfono móvil para la realización de la práctica

En el curso 2014-15, varios grupos de alumnos han realizado esta práctica mediante el acelerómetro incorporado en el teléfono, registrando la aceleración en función del tiempo.

A partir de una encuesta de satisfacción realizada a los alumnos, hemos observado una calificación mucho más alta en todos los apartados de la encuesta para los grupos que han 
realizado la práctica con el smartphone, a excepción de una pregunta relacionada con el tiempo disponible para la realización de la práctica.

Desde nuestro punto de vista, el problema del tiempo tiene una fácil explicación. La utilización del smartphone necesita la instalación de la aplicación, y que los alumnos se familiaricen con su utilización. Además, los alumnos también necesitan más tiempo para el tratamiento de los datos: tienen que hacer una serie de operaciones con los datos, para luego utilizar una plantilla EXCEL suministrada para el cálculo de la pulsación. Todo ello requiere más tiempo que con en el método tradicional, donde los alumnos tan solo tienen que cronometrar el tiempo necesario en realizar una serie de oscilaciones.

No obstante, los profesores de la asignatura consideramos que este método permite entender mejor el fenómeno físico estudiado, porque los alumnos pueden visualizar la aceleración del movimiento armónico simple estudiado, y no se limitan tan solo a cronometrar oscilaciones.

\section{Momento de inercia}

En la práctica del momento de inercia, los alumnos disponen de una plataforma giratoria y un sistema de poleas, de modo que al dejar caer una pesa, mediante el sistema de poleas, comienza a girar la plataforma. Midiendo el tiempo de caída de la pesa, los alumnos determinan su aceleración lineal, la aceleración angular de la plataforma y finalmente el momento de inercia de la plataforma.

Mediante el acelerómetro del dispositivo móvil, los alumnos han medido la aceleración centrífuga durante el movimiento giratorio del disco, y a partir de ella han determinado la aceleración angular del disco. En la figura 3 se muestra el dispositivo experimental para la realización de la práctica, y en la figura 4 un ejemplo de medida realizada con el Smartphone. 


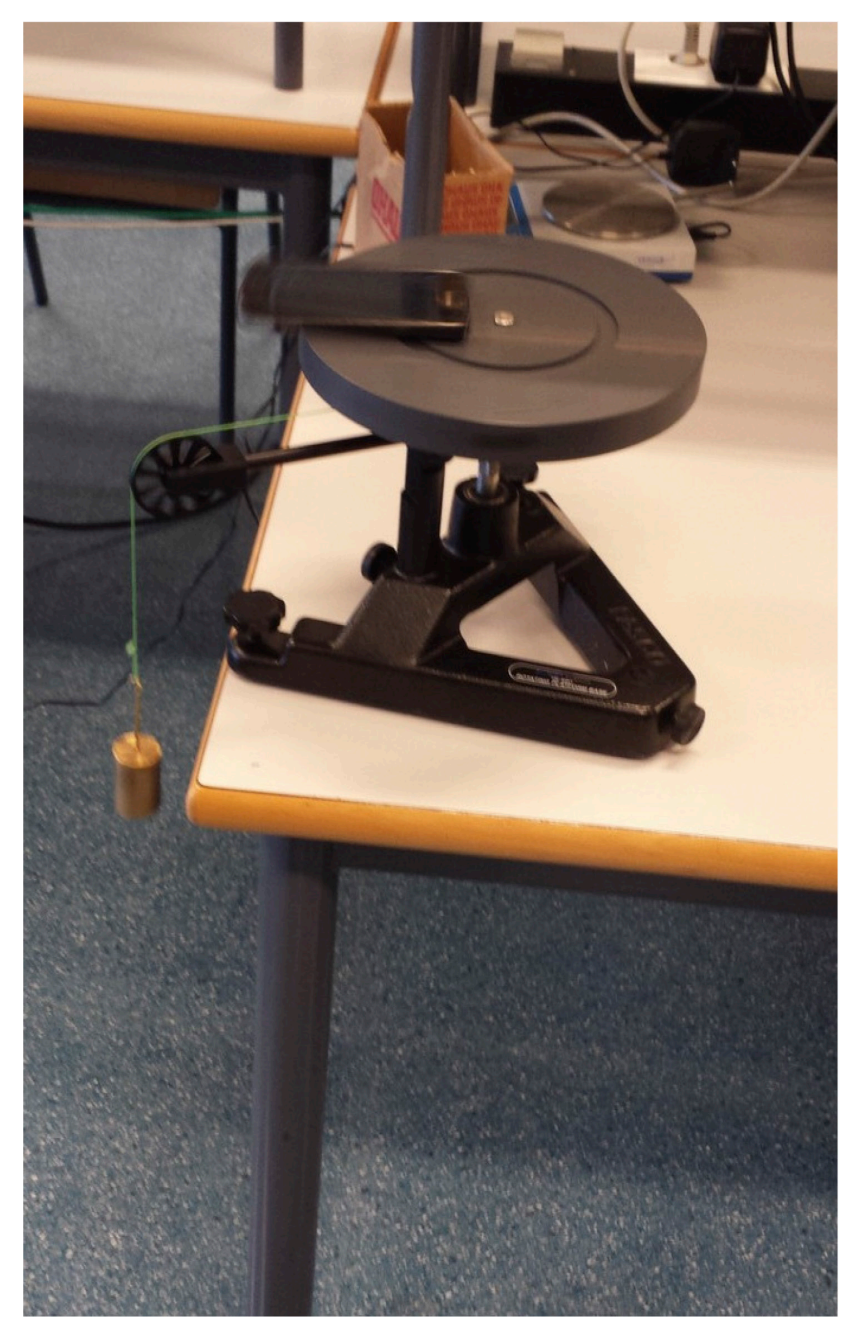

Fig. 3 Colocación del teléfono móvil para la realización de la práctica

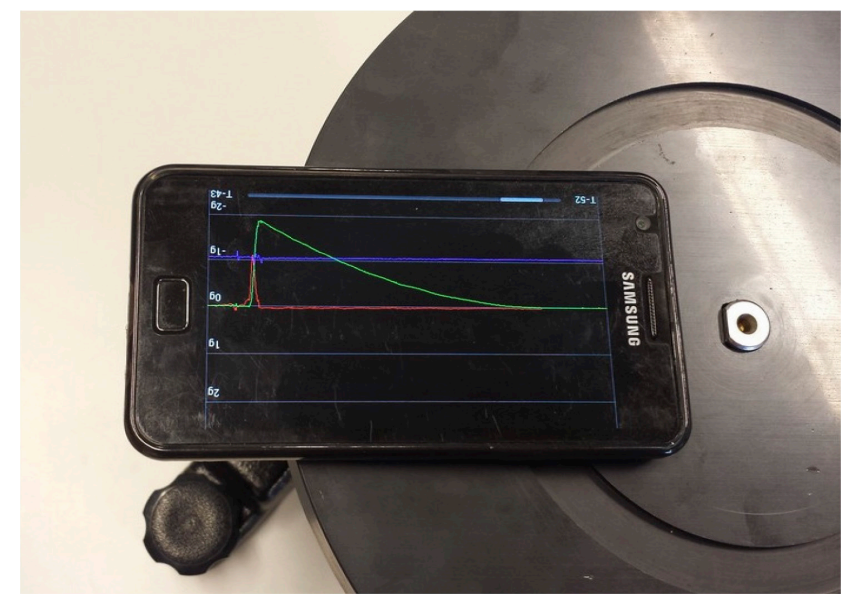

Fig. 4 Detalle de la medida de la aceleración en el Smartphone.

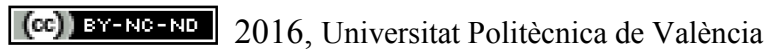

Congreso In-Red (2016) 


\section{Efecto Doppler}

Hemos desarrollado dos aplicaciones para Android ${ }^{\mathrm{TM}}$ capaces de medir la frecuencia de un sonido con gran precisión, y registrar dicha medida como función del tiempo: Analizador de frecuencia y Efecto Doppler (Gómez-Tejedor, Castro-Palacio y Monsoriu 2014; GómezTejedor y Monsoriu 2014).

Mediante dicha aplicación se puede medir el cambio de frecuencia del sonido debido al movimiento del observador (el smartphone en este caso).

Además, dado que la frecuencia observada depende de la velocidad, se puede determinar la velocidad del dispositivo móvil, y estudiar así diferentes tipos de Movimiento Rectilíneo: Uniforme, Uniformemente Acelerado, Armónico Simple, Oscilatorio Amortiguado, etc.

Una de las grandes ventajas de esta propuesta es su bajo coste. En este sentido, un montaje de la marca Phywe para la medida del efecto Doppler en ondas acústicas tiene un precio de 3.182,59 €. En este caso, el único material que necesitamos es un altavoz, cuyo coste es bastante reducido, el smartphone del alumno, y un carril cinemático del que se dispone habitualmente en el Laboratorio de física, ya que se utiliza para diversas prácticas de laboratorio.

En la figura 5 se puede observar una imagen del dispositivo experimental, en el cual se sitúa un altavoz en el carrito del carril cinemático, y el alumno mide la frecuencia mediante su teléfono móvil.

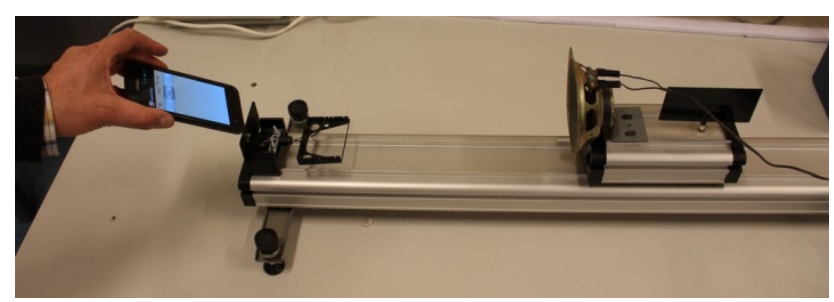

Fig. 5 Montaje experimental para la medida del efecto Doppler.

\section{Batido acústico}

En este caso, siguiendo con la utilización del micrófono del smartphone como sensor, se pretende diseñar una práctica de superposición de ondas (batido acústico) en la que junto a elementos habituales dentro de un laboratorio de física, como son los generadores de corriente y los altavoces, se incluye el smartphone como herramienta de medida (Giménez Valentín et al. 2015). A través de una sencilla aplicación gratuita de Android ${ }^{\mathrm{TM}}$, que mide la intensidad acústica en función del tiempo y permite exportar los datos a una hoja de cálculo, se puede obtener la frecuencia del batido generado por la superposición de dos ondas acústicas sinusoidales de igual intensidad y de frecuencias próximas y comprobar que la diferencia entre estas frecuencias coincide con la de dicho batido (Figura 6). 


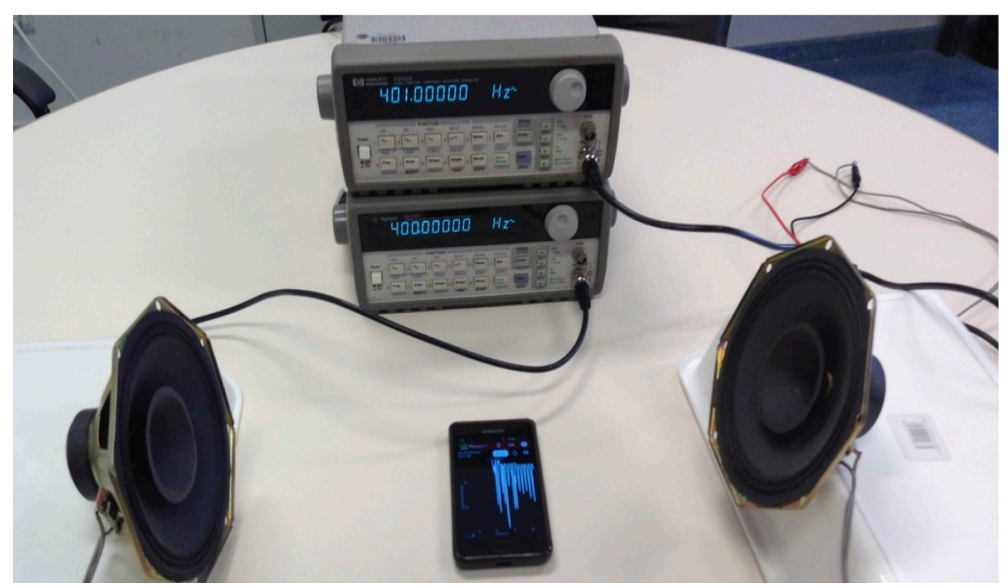

Fig. 6 Montaje experimental para la medida del batido acústico.

\section{Curva de Lissajous}

En un proyecto final de carrera, dirigido por uno de los profesores miembros del equipo de trabajo, se ha desarrollado en el laboratorio de física una pequeña mesa de aire, en la cual se pueden estudiar movimientos en dos dimensiones. Dicha mesa de aire dispone de una plataforma sobre la cual se puede situar el smartphone. Dicha plataforma se sujeta mediante muelles a los bordes de la mesa, permitiendo realizar oscilaciones en los ejes X e Y. (figura 7) Registrando la aceleración del dispositivo, se pueden obtener diferentes figuras de Lissajous, dependiendo de las constantes elásticas de los muelles utilizados en ambos ejes.

De nuevo, para tener una idea del ahorro que esta práctica puede suponer para el laboratorio, podemos mencionar que un montaje comercial de la marca 3B Scientific para la realización de esta práctica tiene un coste unitario de 3.041,94€. 
(a)

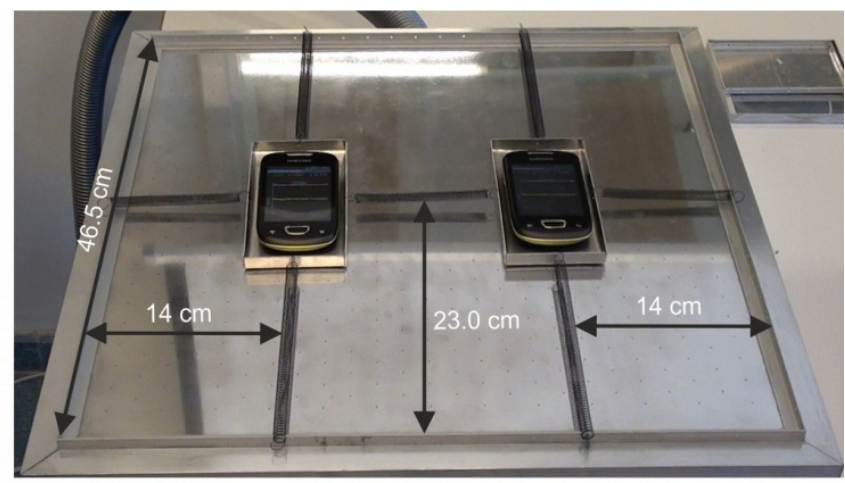

(b)

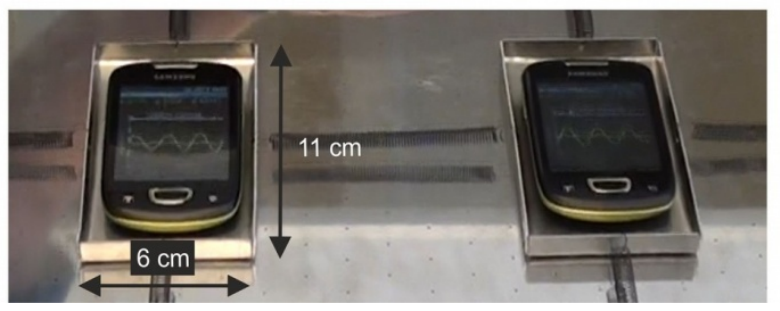

Fig. 7. Montaje experimental para obtener las curvas de Lissajous.

\section{Resultados}

Para evaluar los objetivos alcanzados, hemos realizado una encuesta de satisfacción a los alumnos. En el caso de la práctica de oscilaciones, como disponíamos de docencia en varios grupos, se ha comparado los resultados de la encuesta en los grupos tradicionales, con los resultados en los grupos donde hemos introducido la innovación. En el caso del momento de inercia, como solamente disponíamos de docencia en un grupo, únicamente hemos realizado la práctica mediante la utilización del smartphone, por lo que no se puede comparar con el método tradicional. En este caso además, hemos añadido una pregunta a la encuesta, la número 10 de la tabla, específica para evaluar si la utilización del teléfono móvil les ha resultado interesante y motivadora. Dado que los primeros resultados obtenidos con los alumnos han sido bastante satisfactorios, para el caso de la práctica del efecto Doppler, todos los alumnos han realizado la práctica mediante la utilización del smartphone.

En la tabla 1 se muestran las preguntas realizadas a los alumnos, así como la valoración realizada en cada grupo de alumnos, en una escala de 0 a 10. La encuesta se ha realizado a un total de 395 alumnos de la ETS de Ingeniería del Diseño durante los cursos 2014-15 y 2015-16, 137 de los cuales han realizado la práctica de oscilaciones de manera tradicional, 132 utilizando el teléfono móvil, 27 han realizado la práctica del momento de inercia, y 99 han realizado la práctica del efecto Doppler. 
Tabla 1. Encuesta realizada a los alumnos, con sus correspondientes resultados para cada uno de los grupos objeto de estudio

\begin{tabular}{|c|c|c|c|c|}
\hline \multirow[b]{2}{*}{ Pregunta } & \multicolumn{2}{|c|}{$\begin{array}{l}\text { Oscilaciones: determinación de } \\
\text { la constante de un muelle }\end{array}$} & \multirow{2}{*}{$\begin{array}{c}\text { Momento de } \\
\text { Inercia } \\
\text { (27 alumnos) }\end{array}$} & \multirow{2}{*}{$\begin{array}{c}\text { Efecto } \\
\text { Doppler } \\
\text { (99 alumnos }\end{array}$} \\
\hline & $\begin{array}{l}\text { Tradicional } \\
\text { (137 alumnos) }\end{array}$ & $\begin{array}{l}\text { Smartphone } \\
\text { (132 alumnos) }\end{array}$ & & \\
\hline $\begin{array}{l}\text { 1. La práctica se adecua a la } \\
\text { temática general de la asignatura }\end{array}$ & 7.6 & 9.0 & 7.2 & 8.7 \\
\hline $\begin{array}{l}\text { 2. Los objetivos a conseguir se } \\
\text { han indicado claramente al inicio } \\
\text { de la práctica }\end{array}$ & 7.1 & 8.3 & 6.8 & 7.6 \\
\hline $\begin{array}{l}\text { 3. El tiempo disponible para } \\
\text { realizar la práctica es adecuado }\end{array}$ & 6.3 & 5.9 & 7.1 & 8.5 \\
\hline $\begin{array}{l}\text { 4. La metodología empleada y las } \\
\text { actividades realizadas son } \\
\text { adecuadas }\end{array}$ & 6.6 & 8.2 & 6.9 & 7.8 \\
\hline $\begin{array}{l}\text { 5. El material utilizado me ha } \\
\text { resultado motivador }\end{array}$ & 5.8 & 8.2 & 7.1 & 7 \\
\hline $\begin{array}{l}\text { 6. Me ha sorprendido } \\
\text { procedimiento de medida }\end{array}$ & 4.9 & 8.1 & 6.7 & 6.8 \\
\hline $\begin{array}{l}\text { 7. El profesor ha resuelto las } \\
\text { dudas con claridad y precisión }\end{array}$ & 6.5 & 8.5 & 6.6 & 7.3 \\
\hline $\begin{array}{l}\text { 8. Esta práctica me ha resultado } \\
\text { más interesante que las anteriores }\end{array}$ & 5.7 & 8.1 & 6.9 & 6.9 \\
\hline $\begin{array}{l}\text { 9. Mi valoración general de la } \\
\text { práctica es buena }\end{array}$ & 6.8 & 8.5 & 5.8 & 7.7 \\
\hline $\begin{array}{l}\text { 10. El método de medida } \\
\text { utilizando el teléfono móvil me ha } \\
\text { parecido interesante y motivador. }\end{array}$ & - & - & 10.0 & 7.8 \\
\hline
\end{tabular}

A la vista de los resultados de la encuesta, claramente se puede observar una calificación muy buena en todos los apartados. En particular, en el caso de la práctica de oscilaciones se observa una calificación mucho más alta en todos los apartados de la encuesta para los grupos que han realizado la práctica con el teléfono móvil comparado con los que han seguido el método tradicional. En este caso, tan solo la pregunta relacionada con el tiempo disponible para la realización de la práctica obtiene una puntuación un poco menor.

Como se ha explicado en la sección anterior, el problema con el tiempo de realización de la práctica se puede asignar al tiempo requerido para la instalación de la aplicación en el teléfono móvil, la familiarización de los alumnos a ésta, el envío de los datos del teléfono móvil al ordenador y el uso de una hoja de cálculo para el análisis de los datos obtenidos. 
Todo ello requiere más tiempo que en el método tradicional, donde los alumnos tan solo tienen que cronometrar el tiempo necesario en realizar una serie de oscilaciones.

No obstante, los profesores de la asignatura consideramos que este método permite entender mejor el fenómeno físico estudiado, porque los alumnos pueden visualizar el movimiento armónico simple estudiado, y no se limitan tan solo a cronometrar oscilaciones.

Para solucionar el problema del tiempo en futuros cursos, tenemos varias propuestas. En primer lugar, pedir a los alumnos que se instalen la aplicación, se familiaricen con ella, y la configuren antes de realizar la sesión práctica en el laboratorio. En segundo lugar, proporcionar a los alumnos una plantilla en Excel, para que resulte más rápido y fácil realizar el tratamiento de los datos.

\section{Conclusiones}

Como conclusiones más relevantes, podemos afirmar que los alumnos se sienten motivados e interesados por la innovación metodológica introducida que supone la utilización del teléfono móvil como dispositivo de medida en el laboratorio de física.

Esta metodología puede ser empleada en cualquier laboratorio de física de primeros cursos de grado universitario, dado que en todos ellos se realizan prácticas muy similares a las descritas en este trabajo, y todos los alumnos disponen de smartphone para la realización de las experiencias.

Asimismo, actualmente la mayoría de estudiantes de Bachillerato disponen también de smartphones, por lo que mediante la metodología introducida en este trabajo se podría plantear también una serie de experiencias de laboratorio para las asignaturas de física de Bachillerato. Esto podría resultar muy interesante, dado que habitualmente los Institutos de Enseñanza Secundaria y Bachillerato disponen de pocos recursos económicos para dotar sus laboratorios, y mediante la utilización del smartphone se pueden plantear una serie de experiencias de física con un coste muy reducido.

\section{Agradecimientos}

Los autores desean agradecer al Instituto de Ciencias de la Educación de la Universitat Politècnica de València (España) el apoyo a los Grupos de Innovación Docente MoMa y eMACAFI y el apoyo financiero a través del proyecto PIME 2015 B18.

\section{Referencias}

ARRIBAS GARDE, E., ESCOBAR GARCÍA, I., SUÁREZ RODRÍGUEZ, C. del P., NÁJERA LÓPEZ, A. y BELÉNDEZ VÁZQUE, A., 2015. Medida del campo magnético de imanes pequeños con un smartphone: una práctica de laboratorio muy económica. En: E. ARRIBAS y A. NÁJERA (eds.), Experiencias de innovación docente en la enseñanza de la Física 
Universitaria [en línea]. 4a Edición. Albacete: Lulu Enterprises, pp. 209-222. ISBN 978-1-32625328-8. Disponible en: http://rua.ua.es/dspace/bitstream/10045/48866/1/Cap\%C3\%ADtulo12AB-2015.pdf.

CASTRO-PALACIO, J.C., VELAZQUEZ, L., GÓMEZ-TEJEDOR, J.A., MANJÓN, F.J. y MONSORIU, J.A., 2014. Using a smartphone acceleration sensor to study uniform and uniformly accelerated circular motions. Revista Brasileira de Ensino de Física [en línea], vol. 36, no. 2, pp. 1-5. [Consulta: 23 julio 2015]. ISSN 1806-1117. DOI 10.1590/S1806$11172014000200015 . \quad$ Disponible http://www.scielo.br/scielo.php?script=sci_arttext\&pid=S1806$11172014000200015 \& \operatorname{lng}=$ en\&nrm=iso\&tlng=en.

CASTRO-PALACIO, J.C., VELÁZQUEZ-ABAD, L., GIMÉNEZ, M.H. y MONSORIU, J.A., 2013. Using a mobile phone acceleration sensor in physics experiments on free and damped harmonic oscillations. American Journal of Physics [en línea], vol. 81, no. 6, pp. 472. [Consulta: 29 enero 2016]. ISSN 00029505. DOI 10.1119/1.4793438. Disponible en: http://scitation.aip.org/content/aapt/journal/ajp/81/6/10.1119/1.4793438.

CUENCA-GOTOR, V., MANJON, F.J., SALINAS, I., GIMENEZ-VALENTIN, M.H., SANS, J.A., MONSORIU, J.A. y GOMEZ-TEJEDOR, J.A., 2015. Experimenta la Física con tu Smartphone: medida de la constante elástica de un muelle. 23 Congreso Universitario de Innovación Educativa en las Enseñanzas Técnicas, Valencia. ISBN: 978-84-606-5611-1. S.1.: s.n., pp. 1143-1154.

GIMÉNEZ VALENTÍN, M.H., SALINAS MARÍN, I., MONSORIU SERRA, J.A., CUENCA GOTOR, V.P., MANJÓN HERRERA, F.J., SANS TRESSERRAS, J.A. y GÓMEZ TEJEDOR, J.A., 2015. El teléfono móvil inteligente: una herramienta para el estudio de la acústica experimental. Libro de Actas IN-RED 2015 - Congreso Nacional de Innovación Educativa y de Docencia en Red [en línea]. S.1.: Editorial Universitat Politècnica de València, pp. 1238-1248. ISBN 9788490483961. DOI 10.4995/INRED2015.2015.1593. Disponible en: http://ocs.editorial.upv.es/index.php/ INRED/INRED2015/paper/view/1593.

GÓMEZ-TEJEDOR, J.A., CASTRO-PALACIO, J.C. y MONSORIU, J.A., 2014. The acoustic Doppler effect applied to the study of linear motions. European Journal of Physics [en línea], vol. 35, no. 2, pp. 025006. [Consulta: 23 julio 2015]. ISSN 0143-0807. DOI 10.1088/01430807/35/2/025006. Disponible en: http://stacks.iop.org/01430807/35/i=2/a=025006? $\mathrm{key}=$ crossref. 1 cee5020b0e81bd39b49b437fd2d6b88.

GÓMEZ-TEJEDOR, J.A. y MONSORIU, J.A., 2014. Analizador de Frecuencia: una nueva aplicación docente para Android. Modelling in Science Education and Learning [en línea], vol. 7, pp. 17. [Consulta: 23 julio 2015]. ISSN 1988-3145. DOI 10.4995/msel.2014.2085. Disponible en: http://polipapers.upv.es/index.php/MSEL/article/view/2085.

GONZÁLEZ, M.Á. y GONZÁLEZ REBOLLO, M.Á., 2015. Physics in your pocket: experimenting and learning with your smartphone [en línea]. 2015. S.1.: s.n. [Consulta: 30 marzo 2016]. Disponible en: http://uvadoc.uva.es:80/handle/10324/11893.

$\begin{array}{cccccr}\text { GOODRICH } & \text { ANDRADE, } & \text { H., } & 1996 . & \text { Understanding } & \text { Rubrics. } \\ \text { http://www.middleweb.com/rubricsHG.html } & \text { [en } & \text { línea]. } & \text { Disponible } & \text { en: }\end{array}$ http://www.middleweb.com/rubricsHG.html.

HUETE, F., ESTEBAN, D., SKOURI, M., SILVA, J.B. da, GONZÁLEZ, M.Á., GOUDJAMI, D. y ROCHADEL, W., 2015. SensorMobile, aplicación Android multilingüe con fines docentes para el acceso a sensores de smartphones [en línea]. 2015. S.1.: s.n. [Consulta: 30 marzo 2016]. Disponible en: http://uvadoc.uva.es:80/handle/10324/11882.

2016, Universitat Politècnica de València

Congreso In-Red (2016) 
KLEIN, P., HIRTH, M., GRÖBER, S., KUHN, J. y MÜLLER, A., 2014. Classical experiments revisited: smartphones and tablet PCs as experimental tools in acoustics and optics. Physics Education [en línea], vol. 49, no. 4, pp. 412-418. ISSN 0031-9120. DOI 10.1088/00319120/49/4/412. Disponible en: http://stacks.iop.org/00319120/49/i=4/a=412?key=crossref.3ae90dcebdfe3d2e2152ed1874a9428f.

KUHN, J. y VOGT, P., 2012. Analyzing spring pendulum phenomena with a smart-phone acceleration sensor. The Physics Teacher [en línea], vol. 50, no. 8, pp. 504. [Consulta: 29 enero 2016]. ISSN 0031921X. DOI 10.1119/1.4758162. Disponible en: http://scitation.aip.org/content/aapt/journal/tpt/50/8/10.1119/1.4758162.

KUHN, J. y VOGT, P., 2013. Applications and examples of experiments with mobile phones and smartphones in physics lessons. Frontiers in Sensors [en línea], vol. 1, no. 4, pp. 67-73. [Consulta: 31 marzo 2016]. ISSN 2327-7297. Disponible en: http://www.seipub.org/fs/paperInfo.aspx?ID=9682.

SANS, J.A., GIMÉNEZ, M.H., SALINAS, I., MANJÓN, F.J., CUENCA-GOTOR, V., MONSORIUSERRA, J.A. y GÓMEZ-TEJEDOR, J.A., 2016. Smartphone for teaching experimental Physics. I10th International Technology, Education and Development Conference, INTED2016, Valencia, Spain. ISBN: 978-84-608-5617-7. S.1.: s.n., pp. 1580-88.

SANS, J.A., MANJÓN, F.J., PEREIRA, A.L.J., GOMEZ-TEJEDOR, J.A. y MONSORIU, J.A., 2013. Oscillations studied with the smartphone ambient light sensor. European Journal of Physics [en línea], vol. 34, no. 6, pp. 1349-1354. [Consulta: 23 julio 2015]. ISSN 0143-0807. DOI 10.1088/0143-0807/34/6/1349. Disponible en: http://stacks.iop.org/0143$0807 / 34 / \mathrm{i}=6 / \mathrm{a}=1349$.

SANS, J.A., MANJÓN, J., CUENCA-GOTOR, V., GIMENÉZ-VALENTÍN, M.H., SALINAS, I., BARREIRO, J.J., MONSORIU, J.A. y GOMEZ-TEJEDOR, J.A., 2015. Smartphone: a new device for teaching Physics. HEAd'15. Conference on Higher Education Advances [en línea]. S.1.: Editorial Universitat Politècnica de València, pp. 415-422. ISBN 9788490483404. DOI 10.4995/HEAd15.2015.332. Disponible en: http://ocs.editorial.upv.es/index.php/ HEAD/HEAD15/paper/view/332.

TUSET-SANCHIS, L., CASTRO-PALACIO, J.C., GÓMEZ-TEJEDOR, J.A., MANJÓN, F.J. y MONSORIU, J.A., 2015. The study of two-dimensional oscillations using a smartphone acceleration sensor: example of Lissajous curves. Physics Education [en línea], vol. 50, no. 5, pp. 580-586. [Consulta: 11 septiembre 2015]. ISSN 0031-9120. DOI 10.1088/00319120/50/5/580. Disponible en: http://iopscience.iop.org/article/10.1088/0031-9120/50/5/580.

UPV VICERRECTORADO DE ESTUDIOS CALIDAD Y ACREDITACIÓN, 2016. Dimensiones Competenciales UPV. http://www.upv.es/contenidos/ICEP/info/DimensionesCompetenciales.pdf [en línea]. Disponible en: http://www.upv.es/contenidos/ICEP/info/DimensionesCompetenciales.pdf. 